This is the author's copy of the publication as archived with the DLR's electronic library at http://elib.dlr.de . Please consult the original publication for citation.

\title{
Cascaded Trajectory Tracking Control for Automated Vehicles
}

R. de Castro and J. Brembeck

This work deals with the design of a trajectory tracking controller (TTC) for highly automated vehicles. The proposed design follows a modular architecture, composed of an inner velocity loop and an outer trajectory tracking loop. The outer loop, which represents the main focus on this work, is further divided into two parts. The first uses input-output linearization methods to tackle model nonlinearities. The second part exploits Lyapunov methods and convex optimization to enforce physical constraints -e.g. friction circle - and minimize TTC's loss of performance. Simulation results demonstrate that, in comparison with unconstrained TTC methods, the proposed approach is able to reduce tracking errors in up to $50 \%$ when the vehicle operates at the limit of friction.

\section{Copyright Notice}

(C2020 IEEE. Personal use of this material is permitted. Permission from IEEE must be obtained for all other uses, in any current or future media, including reprinting/republishing this material for advertising or promotional purposes, creating new collective works, for resale or redistribution to servers or lists, or reuse of any copyrighted component of this work in other works.

R. de Castro and J. Brembeck, " Cascaded Trajectory Tracking Control for Automated Vehicles," 2019 IEEE 90th Vehicular Technology Conference (VTC2019-Fall) 10.1109/VTCFall.2019.8891467 


\title{
Cascaded Trajectory Tracking Control for Automated Vehicles
}

\author{
Ricardo de Castro, Jonathan Brembeck \\ Institute of System Dynamics and Control, German Aerospace Center (DLR) \\ Muenchener Str. 20, 82234 Wessling, Germany \\ Email: Ricardo.deCastro@dlr.de
}

\begin{abstract}
This work deals with the design of a trajectory tracking controller (TTC) for highly automated vehicles. The proposed design follows a modular architecture, composed of an inner velocity loop and an outer trajectory tracking loop. The outer loop, which represents the main focus on this work, is further divided into two parts. The first uses input-output linearization methods to tackle model nonlinearities. The second part exploits Lyapunov methods and convex optimization to enforce physical constraints - e.g. friction circle - and minimize TTC's loss of performance. Simulation results demonstrate that, in comparison with unconstrained TTC methods, the proposed approach is able to reduce tracking errors in up to $50 \%$ when the vehicle operates at the limit of friction.
\end{abstract}

\section{INTRODUCTION}

Automated driving is currently envisaged as a key technology for the future of road transportation. As discussed in [1], automated driving has the potential to decrease road accidents due to human errors, improve traffic flow, energy efficiency and passenger comfort. For the successful deployment of this technology several technical areas need to be tackled, including reliable perception of the environment, safe trajectory generation and accurate vehicle control. This work focuses on the vehicle control, with particular attention to the design of the trajectory tracking controller (TTC). From a control architecture perspective, there are two main design avenues that can be explored to develop TTCs. The first, called integrated architecture, implements the TTC using a single controller. The second, called cascaded architecture, splits the problem into two loops (see Fig. 1). The inner loop manipulates the vehicle actuators -steering, braking and traction motor- in order to track longitudinal velocity and yawrate references, while fulfilling vehicle stability limits. The outer loop generates velocity setpoints in order to execute the reference trajectory, computed by the trajectory planner.

The main goal of this work consists in designing the outer loop of the TTC. In the literature, several solutions can be found for this design problem. They range from nonlinear control laws, reliant on Lyapunov methods [2], input-output linearization [3], linear control based on disturbance observers (DOB) [4], sliding mode control [5] and model-predictive control (MPC) [6]. The majority of approaches usually assume that the vehicle operates with negligible wheel slips and/or distant from friction limits. In automotive applications these assumptions are not always easy to fulfill, especially when performing emergency maneuvers, such as obstacle avoidance

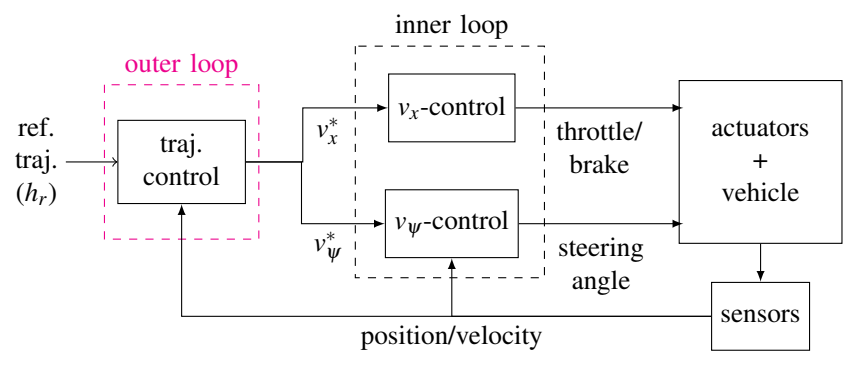

Fig. 1. Block diagram of the cascaded trajectory tracking controller (notation: $v_{x}^{*}=$ longitudinal velocity reference; $v_{\psi}^{*}=$ yaw-rate reference, $h_{r}=$ reference trajectory.)

with aggressive braking and cornering accelerations. If the TTC's outer loop neglects these physical limits, infeasible references might be requested to the inner loops, which - as will be shown in later sections - might increase trajectory tracking errors and compromise the safe execution of planned maneuvers. Integrating physical constraints into the design of the TTC's outer loop is, however, a challenging task. This is due to nonlinear coupling between longitudinal and lateral tire force constraints - also known as friction circle [7] - and the dependence on tire-road friction coefficient, which is timevarying and usually uncertain.

Spurred by these challenges, we propose a novel method to handle physical constraints in the design of the TTC's outer loop. The key idea is to augment a nominal control law -designed without explicit consideration of constraintswith a corrective control action that simultaneously enforces physical constraints and minimizes loss of performance when the vehicle operates close to friction limits. This augmentation relies on the combination of Lyapunov methods [8] and convex optimization methods [9] and represents the main contribution of the present manuscript.

\section{Model And Problem Formulation}

This section provides the control-oriented model for the design of the TTC's outer loop, including physical actuation constraints, and the control problem formulation.

\section{A. Vehicle Model}

Let us consider the three degree-of-freedom planar model of the vehicle (see Fig. 2). In this model, $\mathbf{p}=\left[\begin{array}{ll}p_{X} & p_{Y}\end{array}\right]^{T}$ is 
the vehicle position represented in the $X Y$ coordinate system fixed with the road surface, and $\psi$ the yaw angle between the $X$ axis and the vehicle's $x$ axis. The vector $\mathbf{v}_{x y}=\left[\begin{array}{ll}v_{x} & v_{y}\end{array}\right]^{T}$ contains the vehicle linear velocity - with $v_{x}$ the longitudinal velocity and $v_{y}$ the lateral velocity, defined in the vehicle's local frame $(x y)$ - while $v_{\psi}$ is the yaw-rate. The mathematical model is described as:

$$
\begin{aligned}
\dot{\mathbf{p}} & =\mathbf{R}(\psi) \mathbf{v}_{x y}, \quad \dot{\psi}=v_{\psi} \\
\mathbf{R}(\psi) & =\left[\begin{array}{cc}
\cos (\psi) & -\sin (\psi) \\
\sin (\psi) & \cos (\psi)
\end{array}\right]
\end{aligned}
$$

As depicted in Fig. 1, the inner loop controls the $v_{x}$ and $v_{\psi}$ velocities. The longitudinal velocity is controlled through manipulation of the powertrain actuators (brakes and traction motor), while the yaw-rate is controlled through a steer-bywire actuator, installed in the vehicle's front axle. Control Lyapunov functions are used to construct these inner loops (see [10] for details). Their dominant response is approximated here with first-order dynamics

$$
\begin{aligned}
\dot{v}_{x} & =\alpha_{x}\left(v_{x}^{*}-v_{x}\right)+d_{x} \\
\dot{v}_{\psi} & =\alpha_{\psi}\left(v_{\psi}^{*}-v_{\psi}\right)+d_{\psi}
\end{aligned}
$$

where $v_{i}^{*}$ is the velocity reference signal transmitted to the inner loop $i=\{x, \psi\}$, and $\alpha_{i}=1 / \tau_{i}$ the inverse of the dominant time constant. The disturbances $d_{x}, d_{\psi}$ capture model mismatches between the real vehicle response and the controloriented model (3). They are assumed to lie in the set $\mathscr{D}=\left\{\left(d_{x}, d_{\psi}\right):\left|d_{i}\right| \leq \bar{d}_{i}, i \in\{x, \psi\}\right\}$, where $\bar{d}_{i}$ is a known bound.

While $v_{x}$ and $v_{\psi}$ can be accurately controlled by the inner loops, the vehicle's lateral velocity $v_{y}$ is usually more difficult to measure and control. In practice, the inner yaw-rate loop contains additional control mechanisms to keep the value of $v_{y}$ small, which ensures that the vehicle remains within its safe operation envelope -see [10] for details. As a result, we will assume that inner loop guarantees $\left|v_{y}\right| \leq \bar{v}_{y}$, where $\bar{v}_{y}$ is a small value. Based on this assumption, the lateral velocity dynamics can be represented as:

$$
\dot{v}_{y}=w
$$

where $w$ is an exogenous signal that can be measured. For example, using the lateral acceleration $\left(a_{y}\right)$ and the velocity signals provided by the vehicle's inertial measure unit (IMU), this exogenous signal can be obtained as $w=a_{y}-v_{x} v_{\psi}$ [11]. To further facilitate the outer-loop design, we will introduce the following virtual control inputs

$$
u_{i}=\alpha_{i}\left(v_{i}^{*}-v_{i}\right), \quad i=\{x, \psi\}
$$

From a physical point of view, $u_{x}$ can be interpreted as a desired linear acceleration and $u_{\psi}$ a desired yaw acceleration. Based on these elements, the combined vehicle planar model and inner control loops can be compactly represented as:

$$
\dot{\mathbf{z}}=\left[\begin{array}{c}
\mathbf{R}(\psi) \mathbf{v}_{x y} \\
v_{\psi} \\
\mathbf{F}_{1} w \\
0
\end{array}\right]+\left[\begin{array}{cc}
\mathbf{0} & \mathbf{0} \\
0 & 0 \\
\mathbf{0} & \mathbf{F}_{2} \\
0 & 1
\end{array}\right](\mathbf{u}+\mathbf{d})
$$

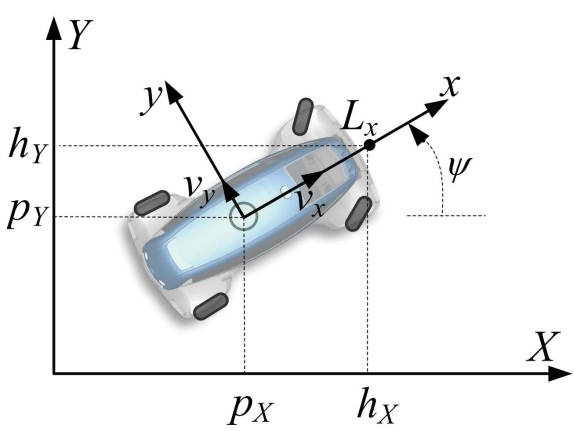

Fig. 2. Planar vehicle model. The point $\left(p_{X}, p_{Y}\right)$ represents the position of the vehicle's center of gravity, while $\left(h_{X}, h_{Y}\right)$ is the virtual point to be tracked.

where $\mathbf{z}=\left[\begin{array}{llll}\mathbf{p}^{T} & \psi & \mathbf{v}_{x y} & v_{\psi}\end{array}\right]^{T}$ is the vehicle state, $\mathbf{u}=$ $\left[\begin{array}{ll}u_{x} & u_{\psi}\end{array}\right]^{T}$ the virtual control input, $\mathbf{d}=\left[\begin{array}{ll}d_{x} & d_{y}\end{array}\right]^{T}$ the disturbances and $\mathbf{0}$ a zero matrix with 2 rows and 1 column. The model also depends on the auxiliary matrices $\mathbf{F}_{1}=\left[\begin{array}{ll}0 & 1\end{array}\right]^{T}$, $\mathbf{F}_{2}=\left[\begin{array}{ll}1 & 0\end{array}\right]^{T}$. It is assumed that the state $\mathbf{z}$ is measured or estimated by the vehicle's on-board sensors.

\section{B. Control Constraints}

The vehicle control is constrained by two types of physical limits. The first type emerges due to constraints in the vehicle actuators, such as limits in braking and traction motor torques. The second type is due to constraints in the friction forces produced by the tires. These constraints can be represented through the vehicle friction circle [7]:

$$
\mathscr{A}=\left\{\left(a_{x}, a_{y}\right): a_{x}^{2}+a_{y}^{2} \leq \mu^{2} g^{2}, \quad \underline{a}_{x} \leq a_{x} \leq \bar{a}_{x}\right\}
$$

where $a_{x}$ is the vehicle's longitudinal acceleration, $a_{y}$ the lateral acceleration, $\mu$ the tire-road adhesion conditionsm, $g$ the gravity acceleration, and $\underline{a}_{x}, \bar{a}_{x}$ represent the minimum and maximal longitudinal acceleration.

The acceleration limits $\mathscr{A}$ are relevant, not only for the inner loop, but also to the TTC's outer loop. If the outer loop neglects these limits, infeasible reference signals might be sent to the inner loops, compromising trajectory tracking performance. To mitigate these risks, the acceleration limits $\mathscr{A}$ will be transformed into a set of admissible control inputs $\mathscr{U}$. More specifically, pragmatic approximations will be used to relate $\left(a_{x}, a_{y}\right)$ with the virtual control inputs $\left(u_{x}, u_{\psi}\right)$. For example, neglecting the disturbances $d_{x}$, the longitudinal acceleration can be approximated as:

$$
a_{x}=\dot{v}_{x}-v_{\psi} v_{y}=u_{x}+d_{x}-v_{\psi} v_{y} \approx u_{x}-v_{\psi} v_{y}
$$

For the lateral acceleration mapping, we assume perfect tracking of the yaw-rate reference $\left(v_{\psi} \approx v_{\psi}^{*}\right)$ and make use of (4),(5) to approximate the lateral acceleration as:

$$
a_{y}=\dot{v}_{y}+v_{\psi} v_{x} \approx w+\left(\frac{u_{\psi}}{\alpha_{\psi}}+v_{\psi}\right) v_{x}
$$


Replacing (8) and (9) into $\mathscr{A}$ leads to the following input constraint set:

$$
\begin{array}{r}
\mathscr{U}=\left\{\left(u_{x}, u_{\psi}\right): \quad \underline{a}_{x} \leq u_{x}-v_{\psi} v_{y} \leq \bar{a}_{x}, \quad \underline{u}_{\psi} \leq u_{\psi} \leq \bar{u}_{\psi}\right. \\
\left.\left(u_{x}-v_{\psi} v_{y}\right)^{2}+\left(w+\left(\frac{u_{\psi}}{\alpha_{\psi}}+v_{\psi}\right) v_{x}\right)^{2} \leq \mu^{2} g^{2}\right\}
\end{array}
$$

which also contains a lower $\left(\underline{u}_{\psi}\right)$ and upper $\left(\bar{u}_{\psi}\right)$ bound on yaw-acceleration.

\section{Formulation of the Control Problem}

The main goal of the outer-loop controller is to track a virtual point $\mathbf{h}$, located at a constant distance $L_{x}$ from the vehicle's center-of-gravity (see Fig. 2)

$$
\mathbf{h}=\left[\begin{array}{ll}
h_{X} & h_{Y}
\end{array}\right]^{T}=\mathbf{p}+\mathbf{R}(\psi) \mathbf{L}
$$

where $\mathbf{L}=\left[\begin{array}{ll}L_{x} & 0\end{array}\right]^{T}$. The control problem consists in designing a feedback law for $\mathbf{u}$ such that the virtual point $\mathbf{h}$ follows, as closely as possible, the reference trajectory $\mathbf{h}_{r}(t): \mathbb{R} \rightarrow \mathbb{R}^{2}$, while fulfilling the input constraint set $\mathscr{U}$. It is assumed that the reference trajectory $\mathbf{h}_{r}$ complies with the model

$$
\dot{\mathbf{h}}_{r}=\left[\begin{array}{c}
\dot{h}_{r, X} \\
\dot{h}_{r, Y}
\end{array}\right]=\left[\begin{array}{l}
v_{r} \cos \left(\psi_{r}\right) \\
v_{r} \sin \left(\psi_{r}\right)
\end{array}\right], \quad \dot{\psi}_{r}=v_{\psi, r}, \quad \dot{v}_{r}=a_{r}
$$

where $v_{r}, v_{\psi, r}$ are the velocities and yaw-rate references, $a_{r}$ represents the reference tangential acceleration and $\psi_{r}$ the reference yaw angle.

\section{Controller Structure}

The control law for the TTC's outer loop is divided into two components :

$$
\mathbf{u}=\mathbf{u}_{N L}+\Delta \mathbf{u}
$$

The first component $\left(\mathbf{u}_{N L}\right)$ is a nominal control law, obtained through input-output linearization [8]. It attempts to cancel the dominant nonlinearities in the model, which - under the assumption of perfect model $(\mathbf{d}=0)$ and unconstrained input set $\left(\mathscr{U}=\mathscr{R}^{2}\right)$ - would be sufficient to fulfill the tracking requirements. The second component $(\Delta \mathbf{u})$ is a correction control action that enforces the input constraints $\mathscr{U}$ with minimum loss of performance.

\section{NOMinal CONTROLlER}

To derive the nominal control law $\mathbf{u}_{N L}$, let us consider the tracking error $\mathbf{e}=\left[\begin{array}{ll}e_{X} & e_{Y}\end{array}\right]^{T}=\mathbf{h}-\mathbf{h}_{r}$. Since this control law depends on input-output linearization [8], it is necessary to first compute the relative degree of the tracking error, i.e., differentiate e until the control input $\mathbf{u}$ explicitly appears in the derivatives of e. Accordingly, computing the first and second time derivative of the tracking error, one obtains:

$$
\begin{aligned}
\ddot{\mathbf{e}}= & \overbrace{\mathbf{T R}(\psi) v_{\psi} \mathbf{v}_{x y}-\mathbf{R}(\psi) \mathbf{L} v_{\psi}^{2}-\ddot{\mathbf{h}}_{r}+\mathbf{R}(\psi) \mathbf{F}_{1} w}^{\mathbf{f}(\mathbf{z}, w)} \\
& +\underbrace{\left[\mathbf{R}(\psi) \mathbf{F}_{2} \mathbf{T R}(\psi) \mathbf{L}\right]}_{\mathbf{G}(\psi)}(\mathbf{u}+\mathbf{d}) \\
= & \mathbf{f}(\mathbf{z}, w)+\mathbf{G}(\psi)(\mathbf{u}+\mathbf{d})
\end{aligned}
$$

where $\mathbf{T}$ is defined in Appendix. This result enables us to conclude that the relative degree ${ }^{1}$ of the tracking error $\mathbf{e}$ is $\left[\begin{array}{ll}2 & 2\end{array}\right]^{T}$ for $L_{x} \neq 0$. When $L_{x}=0$, the virtual point is located at the vehicle's center of gravity and the matrix $\mathbf{G}(\psi)$ becomes singular $\left(\operatorname{det}(\mathbf{G}(\psi))=L_{x}=0\right)$, which prevents the cancellation of the model nonlinearities.

Let us know consider the following change of variable:

$$
\eta=\left[\begin{array}{c}
\eta_{1} \\
\eta_{2}
\end{array}\right]=\left[\begin{array}{c}
\psi-\psi_{r} \\
v_{y}
\end{array}\right], \quad \zeta=\left[\begin{array}{llll}
\zeta_{1} & \zeta_{2} & \zeta_{3} & \zeta_{4}
\end{array}\right]^{T}=\left[\begin{array}{c}
\mathbf{e} \\
\dot{\mathbf{e}}
\end{array}\right]
$$

which leads to the following dynamics:

$$
\begin{aligned}
\dot{\eta}_{1} & =l(\eta, \zeta), \quad \dot{\eta}_{2}=w \\
\dot{\zeta} & =\mathbf{A} \zeta+\mathbf{B}(\mathbf{f}(\mathbf{z}, w)+\mathbf{G}(\psi)(\mathbf{u}+\mathbf{d}))
\end{aligned}
$$

where $\mathbf{A}, \mathbf{B}$ and $l($.$) are defined in Appendix. In this rep-$ resentation, the system is separated into an internal state $\eta=\left[\begin{array}{ll}\eta_{1} & \eta_{2}\end{array}\right]^{T}$ (not directly controllable by the TTC) and an external state $\zeta$, composed of the tracking error and its time derivatives. By driving the external state $\zeta$ toward the origin, the TTC's control goal is fulfilled. This can be achieved by adopting the following control law:

$$
\mathbf{u}_{N L}=-\mathbf{G}^{-1}(\psi) \mathbf{f}(\mathbf{z}, w)-\mathbf{G}^{-1}(\psi) \mathbf{K} \zeta
$$

where $\mathbf{K} \in \mathbb{R}^{2 \times 4}$ is a matrix selected by the designer such that $(\mathbf{A}-\mathbf{B K})$ is Hurwitz, e.g. using pole-placement methods [8]. Furthermore, it can also be shown that the zero-dynamics $\eta$, in particular the yaw-angle error, are bounded (omitted due to space constraints).

\section{Augmented Controller}

The design of the nominal control law (16) does not take into account the input constraint set $\mathscr{U}$. If the virtual control is not feasible, i.e. $\mathbf{u}_{N L} \notin \mathscr{U}$, then a correction term $\Delta \mathbf{u}$ needs to be incorporated into the control law in order to enforce the input constraint set. However, this correction term might have a negative impact in the performance and stability of the nominal controller. To attenuate these risks, we propose in this section a systematic method to compute the correction term that can simultaneously enforce input constraints $\left(\mathbf{u}_{N L}+\Delta \mathbf{u} \in\right.$ $\mathscr{U}$ ) and minimize loss of performance. The combination of the nominal control law and the corrective term is referred hereafter as the augmented controller.

\section{A. Loss of Performance}

To better understand the loss of performance due to control constraints, let us treat the corrective term $\Delta \mathbf{u}$ as a perturbation and investigate its effect in the closed-loop system using Lyapunov methods. Inserting (16) into (12) leads to the following closed-loop dynamics:

$$
\begin{aligned}
\dot{\zeta} & =(\mathbf{A}-\mathbf{B K}) \zeta+\underbrace{\mathbf{B E}\left(\eta_{1}+\psi_{r}\right)}_{\mathbf{g}(\eta)}(\Delta \mathbf{u}+\mathbf{d}) \\
& =(\mathbf{A}-\mathbf{B K}) \zeta+\mathbf{g}(\eta)(\Delta \mathbf{u}+\mathbf{d})
\end{aligned}
$$

${ }^{1}$ recall that $\mathbf{e}$ has a dimensionally of 2 , consequently the relative degree has to be computed for all elements in $\mathbf{e}$ 
Under undisturbed $(\mathbf{d}=0)$ and unconstrained $(\Delta \mathbf{u}=0)$ conditions, the previous dynamics is dominated by the linear term $(\mathbf{A}-\mathbf{B K}) \zeta$. Thus, closed-loop stability can be investigated through the quadratic Lyapunov function $V(\zeta)=\zeta^{T} \mathbf{P} \zeta$, where $\mathbf{P}$ is a positive definite matrix ${ }^{2}$. Computing the time derivative of $V$ one obtains

$$
\dot{V}\left(\zeta, \Delta_{\dot{V}}, \mathbf{d}\right)=-\zeta^{T} \mathbf{Q} \zeta+2 \zeta^{T} \mathbf{P g}(\eta) \mathbf{d}+\Delta_{\dot{V}}
$$

where $\Delta_{\dot{V}}=2 \zeta^{T} \mathbf{P g}(\eta) \Delta \mathbf{u}$ represents a perturbation introduced by the correction term.

Recall that, ultimate boundedness of $\zeta$, i.e. convergence of $\zeta$ to a small neighborhood around the origin, is achieved if $\dot{V}$ fulfills [8]

$$
\dot{V}\left(\zeta, \Delta_{\dot{V}}, \mathbf{d}\right) \leq-W_{V}(\zeta)<0, \quad \forall\|\zeta\| \geq \varepsilon_{\zeta}, \mathbf{d} \in \mathscr{D}
$$

where $W_{V}(\eta)$ is a positive definite function and $\varepsilon_{\zeta}>0$ a small positive constant that affects the size of the ultimate bound. It can be verified that the nominal control law, with input constraints inactive $\left(\Delta_{\dot{V}}=0\right)$, fulfills condition (20) - see [12] for details. On the other hand, the perturbation introduced by the correction term, $\Delta_{\dot{V}}$, can have a "good" or a "bad" effect in the ultimate boundedness condition. For example, a "good" effect is obtained when $\Delta_{\dot{V}}<0$, which makes $\dot{V}$ more negative, helping the fulfillment of (20). "Bad" effects occur when $\Delta_{\dot{V}}>$ 0 . In this case, $\varepsilon_{\zeta}$ might need to be increased in order to fulfill (20), leading to higher ultimate bounds and - in the worst case scenario - compromise the closed-loop stability.

\section{B. Optimal Correction Term}

In light of the above discussion, the correction term $\Delta \mathbf{u}$ should be selected in order to avoid positive values of $\Delta_{\dot{V}}$. With this goal in mind, we start by upper bounding the perturbation $\Delta_{\dot{V}}$ with a non-negative constant $s$, i.e.

$$
\Delta_{\dot{V}}=2 \zeta^{T} \mathbf{P g}(\eta) \Delta \mathbf{u} \leq s, \quad s \geq 0
$$

From a practical perspective, $s$ can be interpreted as Lyapunovbased performance loss. When $s=0$, no perturbation is introduced in the Lyapunov stability condition and no performance loss occurs. On the other hand, when $s>0$, "bad" perturbation are induced, which might lead to tracking performance loss. The design of the correction term can now be re-phrased as: find $\Delta \mathbf{u}$ that minimizes $s$, while complying with the input constraint set $\mathscr{U}$. This can be translated into the following optimization problem:

$$
\begin{array}{ll}
\min _{\Delta \mathbf{u}, s} & w_{s} s^{2}+\Delta \mathbf{u}^{T} \mathbf{W}_{u} \Delta \mathbf{u} \\
\text { s.t. } & 2 \zeta^{T} \mathbf{P g}(\eta) \Delta \mathbf{u} \leq s \\
& s \geq 0 \\
& {\left[u_{x} \quad u_{\psi}\right]^{T}=\mathbf{u}_{N L}+\Delta \mathbf{u}} \\
& \underline{a}_{x} \leq u_{x}-v_{\psi} v_{y} \leq \bar{a}_{x}, \quad \underline{u}_{\psi} \leq u_{\psi} \leq \bar{u}_{\psi} \\
& \left(u_{x}-v_{\psi} v_{y}\right)^{2}+\left(w+\left(\frac{u_{\psi}}{\alpha_{\psi}}+v_{\psi}\right) v_{x}\right)^{2} \leq \mu^{2} g^{2}
\end{array}
$$

\footnotetext{
${ }^{2}$ obtained by solving the equation $\mathbf{P}(\mathbf{A}-\mathbf{B K})+(\mathbf{A}-\mathbf{B K})^{T} \mathbf{P}=-\mathbf{Q}$, with $\mathbf{Q}$ a positive definite matrix defined by the designer
}
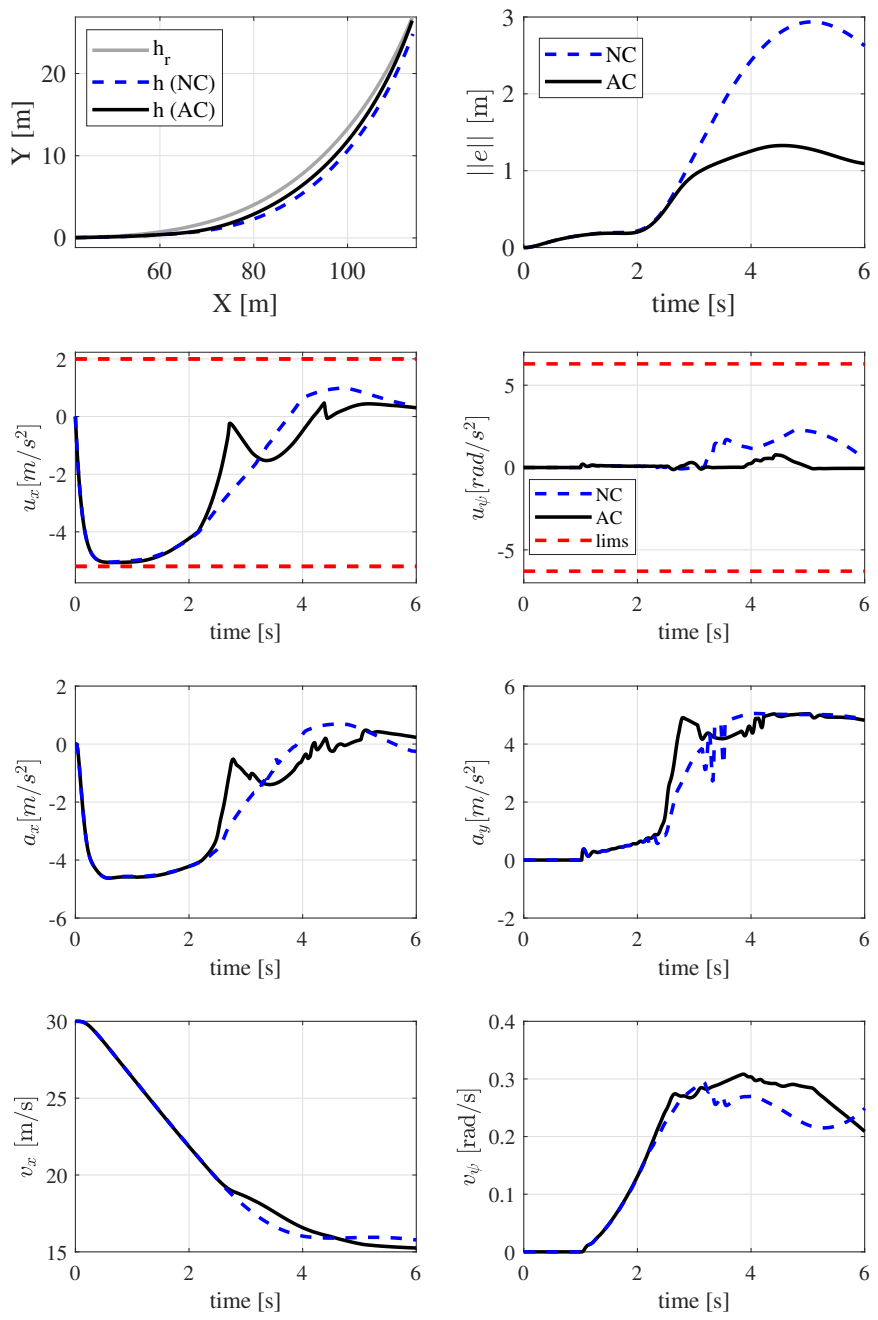

Fig. 3. Simulation results of the TTCs ( $N C=$ nominal controller; $A C=$ augmented controller; red dashed lines represent range-limits in the linear and yaw acceleration).

The above cost function, besides penalizing loss of performance $\left(s^{2}\right)$ also allows minimization of magnitude of the corrective term $(\Delta \mathbf{u})$; trade-off between these two terms is weighted by scalar $w_{s}$ and matrix $\mathbf{W}_{u}$, which are defined by the designer.

\section{VALIDATION}

The trajectory controller was validated through several simulations carried out in a co-simulation environment between Matlab/Simulink and Dymola. The former is responsible for the controller's implementation, while the latter provides a nonlinear double track model, with 14 degree-of-freedom, of the vehicle dynamics model. The vehicle model was implemented in Modelica and parameterized with experimental data of a Volkswagen Passat B6, adapted with a steer-by-wire actuator [13].

Two trajectory controllers were simulated. The first, called nominal controller $(N C)$, relies on the nonlinear control 


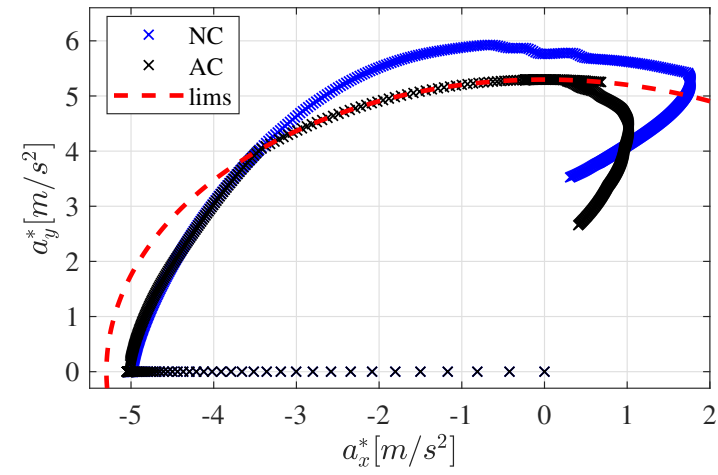

Fig. 4. Acceleration requested by the TTC's outer loop ( $N C=$ nominal controller without saturation; $A C=$ augmented controller); $a_{x}^{*}$ and $a_{y}^{*}$ are computed using the mapping (8),(9); the red dashed line represents the friction circle constraint (7)).

law (16), which is posteriorly saturated ${ }^{3}$ to enforce input constraint $\mathscr{D}$. The second controller, called augmented controller $(A C)$, employs the optimization-based method described in (22) and solved with the embedded conic solver (ECOS) [14]. In both cases, the inner-loop controller relies on input-output methods, combined with control barrier functions, described in [10]. Fig. 3 shows the simulation results of the trajectory controllers obtained while negotiating a left-handed corner with a combined maximum acceleration of $0.45 \mathrm{~g}$ and a known friction coefficient of $\mu=0.55$. The analysis of this maneuver can be divided into two parts. In the first part $(t \in[0,2.5] s)$ the vehicle brakes aggressively in straight line; since the requested accelerations are feasible, both $N C$ and $A C$ have similar responses (see $u_{x}, u_{y}$ signals). In the second part of the maneuver $(t \in[2.5,4] \mathrm{s})$ the vehicle transits from full braking $\left(a_{x}, a_{y}\right)=(-4.5,0) \mathrm{m} / \mathrm{s}^{2}$ to full cornering $\left(a_{x}, a_{y}\right)=(0,4.5) \mathrm{m} / \mathrm{s}^{2}$, demanding vehicle control close to friction limits with combined braking and cornering. The $N C$, because of its disregard for friction limits at design stage, generates an aggressive yaw-rate acceleration $\left(u_{\psi}\right)$ as well as excessive braking demand $\left(u_{x}\right)$, which introduces a significant deviation from the reference trajectory (maximum error $\|\mathbf{e}\|$ of $3 \mathrm{~m})$. These infeasible control requests are also visible in Fig. 4, which depicts the acceleration references generated by the $N C$ (before saturation). On the other hand, the $A C$ provides a more coordinated control; it reduces the amount of braking around $3 \mathrm{~s}$, which then allows a quicker build-up of lateral acceleration $\left(a_{y}\right)$ and yaw-rate $\left(v_{\psi}\right)$. As a result of this intervention, the $A N C$ reduces the maximum tracking error $\|\mathbf{e}\|$ from $3 \mathrm{~m}$ to $1.3 \mathrm{~m}$, i.e. a $56 \%$ reduction.

\section{CONCLUSION}

A two-loop trajectory tracking controller, composed of inner-velocity and outer-position loops, was developed. Particular focus was given to the design of the outer loop in the presence of actuation and friction limits. We have shown how unconstrained control laws, based on input-output linearization, can be augmented with corrective actions that fulfill control constraints and minimize loss of performance. The key idea was to treat the correction actions as perturbations in a Lyapunov sense, which then allowed us to relate corrective actions with Lyapunov-based performance loss. Simulation results demonstrate that, in comparison with unconstrained trajectory tracking controllers, the proposed control method is able to decrease the tracking errors in up to $1.7 \mathrm{~m}$ when the vehicle operates close to friction limits.

\section{APPENDIX I - DEFINITION OF VARIABLES}

The control-oriented model of the vehicle uses the following variables

$$
\mathbf{A}=\left[\begin{array}{llll}
0 & 0 & 1 & 0 \\
0 & 0 & 0 & 1 \\
0 & 0 & 0 & 0 \\
0 & 0 & 0 & 0
\end{array}\right], \quad \mathbf{B}=\left[\begin{array}{ll}
0 & 0 \\
0 & 0 \\
1 & 0 \\
0 & 1
\end{array}\right], \quad \mathbf{T}=\left[\begin{array}{cc}
0 & -1 \\
1 & 0
\end{array}\right]
$$

$$
\begin{aligned}
l(\eta, \zeta)=-\frac{1}{L_{x}}( & \eta_{2}+\sin \left(\eta_{1}+\psi_{r}\right)\left(\dot{h}_{r, X}+\zeta_{3}\right) \\
& \left.-\cos \left(\eta_{1}+\psi_{r}\right)\left(\dot{h}_{r, Y}+\zeta_{4}\right)\right)-v_{\psi, r}
\end{aligned}
$$

\section{REFERENCES}

[1] ERTRAC, "Automated Driving Roadmap," European Road Transport Research Advisory Council, Tech. Rep., 2017.

[2] C. B. Low, "Design, implementation, and experimental validation of a cascaded trajectory tracking controller for nonholonomic car-like wheeled mobile robots with velocity and steering controllers in the loops," in 2017 IEEE Conference on Control Technology and Applications (CCTA), Aug. 2017, pp. 1452-1459.

[3] J. Verhaegh, J. Ploeg, E. v. Nunen, and A. Teerhuis, "Integrated trajectory control and collision avoidance for automated driving," in IEEE International Conference on Models and Technologies for Intelligent Transportation Systems, Jun. 2017, pp. 116-121.

[4] C. Rathgeber, F. Winkler, D. Odenthal, and S. Müller, "Lateral trajectory tracking control for autonomous vehicles," in European Control Conference (ECC), Jun. 2014, pp. 1024-1029.

[5] R. de Castro, M. Tanelli, R. E. Araujo, and S. M. Savaresi, "MinimumTime Path-Following for Highly Redundant Electric Vehicles," IEEE Transactions on Control Systems Technology, vol. 24, no. 2, pp. 487501, Mar. 2016.

[6] X. Qian, A. d. L. Fortelle, and F. Moutarde, "A hierarchical Model Predictive Control framework for on-road formation control of autonomous vehicles," in IEEE Intelligent Vehicles Symposium (IV), Jun. 2016, pp. 376-381.

[7] W. F. M. Milliken and D. L. Milliken, Race Car Vehicle Dynamics. SAE International, 1994.

[8] H. Khalil, Nonlinear Control, 3rd ed. Prentice Hall, 2002

[9] S. Boyd and L. Vandenberghe, Convex optimization, 18th ed. Cambridge: Cambridge Univ. Press, 2015.

[10] R. de Castro and J. Brembeck, "A Control Barrier Function Approach for the Robust Lateral Control of Steer-by-Wire Vehicles," in IEEE Vehicle Power and Propulsion Conference (VPPC), Dec. 2017, pp. 1-6.

[11] R. Rajamani, Vehicle dynamics and control. New York, NY: Springer, 2006.

[12] R. de Castro, "FIF: M6300.04 Entwurf von TFR ist simulativ validiert inkl. Modellierung der maximalen Reglerabweichungen," Deutsches Zentrum für Luft- und Raumfahrt (DLR), Oberpfaffenhofen, Tech. Rep., 2017.

[13] J. Schomerus, M. Gutknecht, P. Luithardt, H. Mosebach, and F. Koster, "Ein Steer-by-Wire-System für hochautomatisierte PKW," in Mechatronik, 2011.

[14] A. Domahidi, E. Chu, and S. Boyd, "ECOS: An SOCP solver for embedded systems," in European Control Conference, 2013.

\footnotetext{
${ }^{3}$ obtained by solving (22) without the stability constraint (22a)
} 